\author{
А.С. Шевельова, А.А. Гриневич \\ Дніпровський наиіональний університет імені Олеся Гончара
}

\title{
ЧИСЕЛЬНО-АНАЛІТИЧНИЙ ПІДХІД ДО ВИЗНАЧЕННЯ ЗОН ПЕРЕДРУЙНУВАННЯ ДЛЯ ТРІЩИНИ НА МЕЖІ ПОДІЛУ П'ЄЗОЕЛЕКТРОМАГНІТНИХ МАТЕРІАЛІВ
}

\begin{abstract}
Розглянута тріщина на межі поділу п'єзоелектромагнітних матеріалів. Використана модель електрично та магнітно проникних умов на берегах тріщини, яка с найбільш адекватною дійсності. Поставлена задача пошуку зон передруйнування, що виникають на продовженнях тріщини. Використано апарат теорії функцій комплексної змінної і отримано наближений чисельно-аналітичний розв'язок, який визначас характеристики вказаних зон. Знайдені залежності довжин зон передруйнування та розкриття тріщини від фізичних характеристик матеріалу і навантаження.

Ключові слова: п'єзоелектромагнітний біматеріал, міжфазна тріщина, електрично та магнітно проникна тріщина, зони передруйнування, проблема Гільберта-Рімана.

\author{
A.E. Sheveleva, A.A. Grynevych
}

Oles Honchar Dnipro National University

\section{NUMERICAL AND ANALYTICAL APPROACH TO DETERMINING OF ELECTRO-MECHANICAL PRE-FRACTURE ZONES FOR AN ELECTRICALLY PERMEABLE INTERFACE CRACK IN A PIEZOELECTROMAGNETIC BIMATERIAL}

Piezoelectromagnetic materials have the ability to serve as sensors and actuators. Therefore, this type of material is used in feedback control systems in robotics, aviation and microelectromechanical systems. But piezoelectric materials are very subjected to fracture, so the study of the problems of deformation of such materials in the presence of cracks is very important. A plane strain problem for two piezoelectromagnetic half-spaces with a crack under at their interface under the action of remote mixed mode mechanical loading magnetic and electrical fluxes is considered. The electrically and magnetically permeable assumption along the crack faces is adopted. According to numerous results this assumption is the most realistic with respect to practice. It is assumed that the substrates are much stiffer than the adhered layer. Therefore, the pre-fracture zones arise at the crack continuations. Normal and shear stresses are assumed to be constant in these zones and must satisfy some material equation, which can be taken from theory or derived experimentally. Modeling the pre-fracture zones by the crack continuations with unknown cohesive stresses on their faces reduces the problem to an elastic interface crack analysis which leads to a Hilbert-Riemann problem. This problem is solved exactly. The pre-fracture zone lengths and stresses in these zones are found from special form algebraical and transcendental equations. These equations are derived from the conditions of stress finiteness at the ends of pre-fracture zones and the material equations. The electrical and magnetic displacements at any point of the pre-fracture zones are found in closed form as well. Numerical

() Шевельова А.С., Гриневич А.А., 2020 
results corresponding to certain material combinations and interlayer material equations are presented. The discussion of these results is also given. It shows particularly that the value of normal stress in the pre-failure zones does not depend on the magnitude of the external load. On the other hand, the length of the pre-fracture zone and the crack opening depends significantly and, moreover, nonlinearly on this parameter. In addition, the values of the length of the pre-fracture zone remain quite small compared to the length of the crack, even for relatively large external stresses. It is important to note that in the suggested model, any singularities connected with the crack are eliminated, i.e., all mechanical and electrical characteristics are limited in the near crack tip region.

Keywords: piezoelectromagnetic bimaterial, interface crack, electrically and magnetically permeable crack, a pre-fracture zone, Hilbert-Riemann problem.

\author{
А.Е. Шевелева, А.А. Гриневич \\ Днипровский национальный университет имени Олеся Гончара
}

\title{
ЧИСЛЕННО-АНАЛИТИЧЕСКИЙ ПОДХОД К ОПРЕДЕЛЕНИЮ ЗОН ПРЕДРАЗРУШЕНИЯ ДЛЯ ТРЕЩИНЫ НА ГРАНИЦЕ РАЗДЕЛА ПЬЕЗОЭЛЕКТРОМАГНИТНЫХ МАТЕРИАЛОВ
}

Рассмотрена трещина на границе раздела пьезоелектромагнитных материалов. Использована модель электрически и магнитно проникающих условий на берегах трещины, которая является наиболее адекватной действительности. Поставлена задача поиска зон предразрушения, возникающих на продолжениях трещины. Использован аппарат теории функций комплексного переменного и получено приближенное численно-аналитическое решение, которое определяет характеристики указанных зон. Найдены зависимости длин зон предразрушения и раскрытия трещины от физических характеристик материала и нагрузки.

Ключевые слова: пьезоэлектромагнитный биматериал, межфазная трещина, электрически и магнитно проницаемая трещина, зоны предразрушения, задача Гильберта-Римана.

Вступ. П'єзоелектромагнітні матеріали мають здатність служити як датчики, так і виконавчі механізми. Тому цей тип матеріалів використовується в системах управління зворотним зв'язком в робототехніці, авіації та в мікроелектромеханічних системах. Але п'єзоматеріали $є$ дуже крихкими, тому вивчення проблем деформування таких матеріалів при наявності в них тріщин є актуальним. Однією з моделей руйнування тіл $з$ тріщинами, що активно використовується на практиці є модель, запропонована в роботах [6, 7]. Один $з$ варіантів іiі розвитку на міжфазну тріщину у п'єзоелектричному біматеріалі був запропонований в роботі [2]. Стосовно п'єзоелектромагнітного біматеріалу аналогічний підхід був розвинутий в роботі [8] для електрично ізольованої та магнітно проникної тріщини. В даній роботі пропонується розвиток моделі зон передруйнування на міжфазні тріщини у п'єзоелектромагнітних біматеріалах для найбільш важливого випадку електрично і магнітно проникної тріщини. 
Постановка задачі та виведення основних співвідношень. Розглядається електрично i магнітно проникна тріщина на межі поділу двох п'єзоелектромагнітних півпросторів.

Основні співвідношення для таких матеріалів мають вигляд:

$$
\begin{gathered}
\left\{\begin{array}{l}
\sigma_{i j}=c_{i j k s} \varepsilon_{k s}-e_{s i j} E_{s}-h_{s i j} H_{s}, \\
D_{i}=e_{i k s} \varepsilon_{k s}+\alpha_{i s} E_{s}+d_{i s} H_{s}, \\
B_{i}=h_{i k s} \varepsilon_{k s}+d_{i s} E_{s}+\gamma_{i s} H_{s},
\end{array}\right. \\
\sigma_{i j, j}=0, D_{i, i}=0, B_{i, i}=0, \varepsilon_{i j}=\frac{1}{2}\left(u_{i, j}+u_{j, i}\right), E_{i}=-\phi_{, i}, H_{i}=-\varphi_{, i},
\end{gathered}
$$

де $D_{i}, B_{i}$ - компоненти векторів електричної та магнітної індукцій; $E_{i}, H_{i}-$ напруженості електричного та магнітного полів; $\phi, \varphi$ - електричний і магнітний потенціали; $c_{i j k s}-$ пружні, $e_{i k s}-$ п'єзоелектричні, $h_{i k s}-$ п’єзомагнітні, $d_{i s}-$ електромагнітні константи, $\alpha_{i s}, \gamma_{i s}$ - діелектричні та магнітні проникності. Для випадку біматеріалу вони використовуються з верхніми індексами (1) і (2) для верхнього та нижнього півпросторів відповідно. Всі поля вважаються такими, що не залежать від координати $x_{2}$.

В роботі [1] показано, що для найбільш важливого для практики трансверсально-ізотропного біматеріалу з віссю симетрії $x_{3}$ при умовах плоскої деформації в площині $\left(x_{1}, x_{3}\right)$ справедливо представлення:

$$
\begin{gathered}
\left\langle\mathbf{V}^{\prime}\left(x_{1}\right)\right\rangle=\mathbf{W}^{+}\left(x_{1}\right)-\mathbf{W}^{-}\left(x_{1}\right), \\
\mathbf{t}^{(1)}\left(x_{1}, 0\right)=\mathbf{G} \mathbf{W}^{+}\left(x_{1}\right)-\overline{\mathbf{G}} \mathbf{W}^{-}\left(x_{1}\right),
\end{gathered}
$$

де $\mathbf{G}$ - матриця розмірністю $4 \times 4$, елементи якої визначаються константами матеріалів, $\mathbf{W}(z)=\left\{W_{1}(z), W_{3}(z), W_{4}(z), W_{5}(z)\right\}^{T}$ - чотирьох-компонентна вектор-функція, яка аналітична всюди, за винятком області тріщини, $\mathbf{W}^{ \pm}\left(x_{1}\right)=\mathbf{W}\left(x_{1} \pm i 0\right),\langle\cdot\rangle$ означає стрибок функції при переході через лінію поділу матеріалів. Вектори $\mathbf{V}$ та $\mathbf{t}$ визначаються так:

$$
\mathbf{V}=\left\{\begin{array}{llll}
u_{1}, & u_{3}, & \varphi, & \phi
\end{array}\right\}^{T}, \mathbf{t}=\left\{\begin{array}{llll}
\sigma_{31}, & \sigma_{33}, & D_{3}, & B_{3}
\end{array}\right\}^{T} .
$$

Оскільки тріщина електрично і магнітно проникна, то $\langle\varphi\rangle=0,\langle\phi\rangle=0$ для $x_{1} \in(-\infty ; \infty)$. Це означає, на основі (2) і (4), що функції $W_{4}(z)$ и $W_{5}(z)$ аналітичні у всій площині, i, значить:

$$
W_{4}(z)=W_{4}^{0}=\text { const }, W_{5}(z)=W_{5}^{0}=\text { const } .
$$

Оскільки для $\left|x_{1}\right| \geq b: \quad \mathbf{W}^{+}\left(x_{1}\right)=\mathbf{W}^{-}\left(x_{1}\right)=\mathbf{W}\left(x_{1}\right), \quad$ то для $\left|x_{1}\right| \geq b \quad 3$ співвідношення (3) отримуємо:

$$
\mathbf{t}^{(1)}\left(x_{1}, 0\right)=(\mathbf{G}-\overline{\mathbf{G}}) \mathbf{W}\left(x_{1}\right) .
$$

Припустимо, що біматеріал знаходиться під дією електричного $D^{\infty}$, магнітного $B^{\infty}$ потоків і механічного навантаження $\sigma^{\infty}, \tau^{\infty}$ на нескінченності. 
Це означає, що

$$
\mathbf{t}^{\infty}=\left\{\sigma^{\infty}, \tau^{\infty}, D^{\infty}, B^{\infty}\right\}=\left.\mathbf{t}^{(1)}\left(x_{1}, 0\right)\right|_{x_{1} \rightarrow \infty}=\left.(\mathbf{G}-\overline{\mathbf{G}}) \mathbf{W}(z)\right|_{z \rightarrow \infty},
$$

а отже

$$
\left.\mathbf{W}(z)\right|_{z \rightarrow \infty}=(\mathbf{G}-\overline{\mathbf{G}})^{-1} \mathbf{t}^{\infty} .
$$

Тобто, потрібні для подальшого аналізу компоненти останнього вектору, які позначимо $W_{4}^{\infty}$ i $W_{5}^{\infty}$, визначаються так

$$
W_{4}^{\infty}=\left\{(\mathbf{G}-\overline{\mathbf{G}})^{-1} \mathbf{t}^{\infty}\right\}_{4}, W_{5}^{\infty}=\left\{(\mathbf{G}-\overline{\mathbf{G}})^{-1} \mathbf{t}^{\infty}\right\}_{5} .
$$

Для розглянутих трансверсально-ізотропних матеріалів з віссю симетрії $x_{3}$ матриця $\mathbf{G}$ має таку структуру:

де усі $g_{i j}$ - дійсні.

$$
\mathbf{G}=\left[\begin{array}{cccc}
i g_{11} & g_{13} & g_{14} & g_{15} \\
g_{31} & i g_{33} & i g_{34} & i g_{35} \\
g_{41} & i g_{43} & i g_{44} & i g_{45} \\
g_{51} & i g_{53} & i g_{54} & i g_{55}
\end{array}\right],
$$

В цьому випадку формули (8) можна представити у формі:

$$
W_{4}^{\infty}=R_{21} \sigma^{\infty}+R_{22} D^{\infty}+R_{23} B^{\infty}, W_{5}^{\infty}=R_{31} \sigma^{\infty}+R_{32} D^{\infty}+R_{33} B^{\infty},
$$

де $R_{i j}$ є елементами матриці $\mathbf{R}=-i \mathbf{S}^{-1}$, причому

$$
\mathbf{S}=\left[\begin{array}{lll}
g_{33} & 2 g_{34} & 2 g_{35} \\
g_{43} & 2 g_{44} & 2 g_{45} \\
g_{53} & 2 g_{54} & 2 g_{55}
\end{array}\right] .
$$

3 урахуванням отриманих подань 3 другого рівняння (2) отримуємо

$$
\begin{gathered}
\sigma_{33}^{(1)}\left(x_{1}, 0\right)+i m_{j} \sigma_{13}^{(1)}\left(x_{1}, 0\right)=\vartheta_{j}\left(F_{j}^{+}\left(x_{1}\right)+\gamma_{j} F_{j}^{-}\left(x_{1}\right)\right)+\sigma_{0}, \\
\left\langle u_{1}^{\prime}\left(x_{1}\right)\right\rangle+i s_{j}\left\langle u_{3}^{\prime}\left(x_{1}\right)\right\rangle=F_{j}^{+}\left(x_{1}\right)-F_{j}^{-}\left(x_{1}\right), \\
D_{3}^{(1)}\left(x_{1}, 0\right)=\left(g_{41}-\frac{g_{43} g_{31}}{g_{33}}\right)\left\langle u_{1}^{\prime}\left(x_{1}\right)\right\rangle+\frac{g_{43}}{g_{33}} \sigma_{33}^{(1)}\left(x_{1}, 0\right)+d_{0}-\frac{g_{43}}{g_{33}} \sigma_{0}, \\
B_{3}^{(1)}\left(x_{1}, 0\right)=\left(g_{51}-\frac{g_{53} g_{31}}{g_{33}}\right)\left\langle u_{1}^{\prime}\left(x_{1}\right)\right\rangle+\frac{g_{53}}{g_{33}} \sigma_{33}^{(1)}\left(x_{1}, 0\right)+b_{0}-\frac{g_{53}}{g_{33}} \sigma_{0},
\end{gathered}
$$

де

$$
\begin{gathered}
F_{j}(z)=W_{1}(z)+i s_{j} W_{3}(z),(j=1,3), \\
m_{1,3}=\mp \sqrt{-\frac{g_{31} g_{33}}{g_{11} g_{13}}}, \gamma_{j}=-\frac{g_{31}+m_{j} g_{11}}{\vartheta_{j}}, \vartheta_{j}=g_{31}-m_{j} g_{11}, s_{j}=\frac{g_{33}+m_{j} g_{13}}{\vartheta_{j}}, \\
\sigma_{0}=2 i\left(g_{34} W_{4}^{\infty}+g_{35} W_{5}^{\infty}\right), d_{0}=2 i\left(g_{44} W_{4}^{\infty}+g_{45} W_{5}^{\infty}\right), b_{0}=2 i\left(g_{54} W_{4}^{\infty}+g_{55} W_{5}^{\infty}\right) .
\end{gathered}
$$


Будемо тепер вважати, що на продовженнях тріщини виникли зони передруйнування $\left[a_{1}, a\right] \mathrm{i}\left[b, b_{1}\right]$, причому напруження в цих зонах постійні. Позначимо їх $\sigma_{33}\left(x_{1}, 0\right)=\sigma^{\prime}, \sigma_{13}\left(x_{1}, 0\right)=\tau^{\prime}, \sigma_{11}\left(x_{1}, 0\right)=\sigma_{1}^{\prime}$ для лівої зони $\mathrm{i}$ $\sigma_{33}\left(x_{1}, 0\right)=\sigma, \sigma_{13}\left(x_{1}, 0\right)=\tau, \sigma_{11}\left(x_{1}, 0\right)=\sigma_{1}$ для правої зони. Значення $\sigma, \tau, \sigma_{1}$ та $\sigma^{\prime}, \tau^{\prime}, \sigma_{1}^{\prime}$ повинні бути визначені. Вважається, що справедливі такі співвідношення для зон передруйнування:

$$
\begin{aligned}
& f\left(\sigma, \tau, \sigma_{1}\right)=0 \text { (права зона), } \\
& f\left(\sigma^{\prime}, \tau^{\prime}, \sigma_{1}^{\prime}\right)=0 \text { (ліва зона). }
\end{aligned}
$$

Функція $f$, яка може бути інтерпретована як деякий закон текучості або руйнування матеріалу прошарку, визначається експериментально або теоретично.

Граничні умови на інтерфейсі можуть бути представлені у вигляді

$$
\begin{gathered}
\langle\mathbf{V}\rangle=0,\langle\mathbf{t}\rangle=0, \text { для } x_{1} \notin\left(a_{1}, b_{1}\right), \\
\sigma_{33}^{(j)}\left(x_{1}, 0\right) \equiv p_{1}\left(x_{1}\right)=\left\{\begin{array}{ll}
\sigma^{\prime}, & a_{1} \leq x_{1} \leq a, \\
\sigma, & b \leq x_{1} \leq b_{1}, \\
0, & a<x_{1}<b,
\end{array} \sigma_{13}^{(j)}\left(x_{1}, 0\right) \equiv p_{2}\left(x_{1}\right)= \begin{cases}\tau^{\prime}, & a_{1} \leq x_{1} \leq a, \\
\tau, & b \leq x_{1} \leq b_{1},(20) \\
0, & a<x_{1}<b .\end{cases} \right.
\end{gathered}
$$

Крім того, $\left\langle\varphi\left(x_{1}\right)\right\rangle=0$ та $\left\langle\phi\left(x_{1}\right)\right\rangle=0$ для $x_{1} \in(-\infty,+\infty)$, оскільки межа поділу матеріалів вважається електрично і магнітно проникною.

Отже, сформульована задача механіки руйнування для тріщини $\left[a_{1}, b_{1}\right]$ між двома півпросторами з невідомими компонентами напружень $\sigma, \tau, \sigma^{\prime}, \tau^{\prime}$ на поверхнях тріщини і невідомими положеннями точок $a_{1}$ та $b_{1}$.

Визначення характеристик зони передруйнування. Для зручності введемо такі нові функції

$$
\Phi_{j}(z)=F_{j}(z)+\Phi_{j}^{0}, \Phi_{j}^{0}=\frac{\sigma_{0}}{\vartheta_{j}\left(1+\gamma_{j}\right)},(j=1,2) .
$$

Співвідношення (11) і (12) приймають таку форму

$$
\begin{gathered}
\sigma_{33}^{(1)}\left(x_{1}, 0\right)+i m_{j} \sigma_{13}^{(1)}\left(x_{1}, 0\right)=\vartheta_{j}\left[\Phi_{j}^{+}\left(x_{1}\right)+\gamma_{j} \Phi_{j}^{-}\left(x_{1}\right)\right], \\
\left\langle u_{1}^{\prime}\left(x_{1}\right)\right\rangle+i s_{j}\left\langle u_{3}^{\prime}\left(x_{1}\right)\right\rangle=\Phi_{j}^{+}\left(x_{1}\right)-\Phi_{j}^{-}\left(x_{1}\right) .
\end{gathered}
$$

Очевидно, що функції $\Phi_{j}(z)$ мають ті ж властивості, що і функції $F_{j}(z)$.

Беручи до уваги, що співвідношення $\Phi_{j}^{+}\left(x_{1}\right)=\Phi_{j}^{-}\left(x_{1}\right) \epsilon$ вірним для $x_{1} \notin\left(a_{1}, b_{1}\right)$, з рівняння (22) випливає, що для $x_{1} \rightarrow \infty$

$$
\vartheta_{j}\left(1+\gamma_{j}\right) \Phi_{j}^{+}\left(x_{1}\right)=\sigma^{\infty}+i m_{j} \tau^{\infty} .
$$

Оскільки функції $\Phi_{j}(z) є$ аналітичні на нескінченності, отримуємо

$$
\left.\Phi_{j}(z)\right|_{z \rightarrow \infty}=\left(\sigma^{\infty}+i m_{j} \tau^{\infty}\right) / r_{j}, r_{j}=\vartheta_{j}\left(1+\gamma_{j}\right) \text {. }
$$


Для подальшого аналізу достатньо використовувати співвідношення (22), (23) i (25) при $j=1$. Ці співвідношення при $j=2$ можуть бути необхідні, тільки коли деякі електромеханічні характеристики знаходяться для точок, розташованих за межами інтерфейсу.

Використовуючи формулу (22) і задовольняючи умовам на інтерфейсі (20), отримуємо

$$
\Phi_{1}^{+}\left(x_{1}\right)+\gamma_{1} \Phi_{j}^{-}\left(x_{1}\right)=p\left(x_{1}\right) / \vartheta_{1} \text { для } x_{1} \in\left(a_{1}, b_{1}\right),
$$

де

$$
p\left(x_{1}\right)=p_{1}\left(x_{1}\right)+i m_{1} p_{2}\left(x_{1}\right) .
$$

Розв'язок задачі (26), відповідно до [4], можна записати у вигляді

$$
\Phi_{1}(z)=\frac{1}{2 \pi i Y(z)}\left[C_{0}+C_{1} z+\frac{1}{\vartheta_{1}} \int_{a_{1}}^{b_{1}} \frac{p(t) Y^{+}(t)}{t-z} d t\right],
$$

де

$$
Y(z)=\left(z-a_{1}\right)^{0,5-i \varepsilon}\left(z-b_{1}\right)^{0,5+i \varepsilon}, \varepsilon=\ln \gamma_{1} / 2 \pi .
$$

Визначаючи $C_{0}$ та $C_{1} 3$ умов на нескінченності і беручи до уваги, що $\Phi_{1}^{+}\left(x_{1}\right)=\Phi_{1}^{-}\left(x_{1}\right)=\Phi_{1}\left(x_{1}\right)$ для $x_{1} \notin\left(a_{1}, b_{1}\right)$ за формулою (22), отримуємо таке співвідношення для напружень на лінії поділу матеріалів

$$
\begin{gathered}
\sigma_{33}^{(1)}\left(x_{1}, 0\right)+i m_{1} \sigma_{13}^{(1)}\left(x_{1}, 0\right)=\frac{1}{2 \pi i Y\left(x_{1}\right)}\left\{2 \pi i\left(x_{1}-\frac{a_{1}+b_{1}}{2}-i \varepsilon l_{1}\right)\left(\sigma^{\infty}+i m_{1} \tau^{\infty}\right)+\right. \\
\left.+\left(1+\gamma_{1}\right)\left[\left(\sigma^{\prime}+i m_{1} \tau^{\prime}\right) \int_{a_{1}}^{a} \frac{Y^{+}(t)}{t-x_{1}} d t+\left(\sigma+i m_{1} \tau\right) \int_{b}^{b_{1}} \frac{Y^{+}(t)}{t-x_{1}} d t\right]\right\}, x_{1}>b_{1}
\end{gathered}
$$

де $l_{1}=b_{1}-a_{1}$.

Далі використовується умова скінченності напружень в точках $a_{1}$ i $b_{1}$. Ця умова виконується, якщо справедливі такі рівняння:

$$
Y\left(\xi_{k}\right)\left[\sigma_{33}^{(1)}\left(\xi_{k}, 0\right)+i m_{1} \sigma_{13}^{(1)}\left(\xi_{k}, 0\right)\right]=0,
$$

де $\xi_{1}=a_{1}, \xi_{2}=b_{1}, k=1,2$.

Рівняння (29) з урахуванням формули (28) та аналогічної формули для $x_{1}<a_{1}$ призводять до такої системи лінійних алгебраїчних рівнянь

$$
\mathbf{N}\left\{\begin{array}{c}
\sigma^{\prime}+i m_{1} \tau^{\prime} \\
\sigma+i m_{1} \tau
\end{array}\right\}=\frac{\pi i l_{1}}{1+\gamma_{1}}\left(\sigma^{\infty}+i m_{1} \tau^{\infty}\right)\left\{\begin{array}{c}
1+2 i \varepsilon \\
-(1-2 i \varepsilon)
\end{array}\right\}
$$

відносно невідомих напружень $\sigma, \tau, \sigma^{\prime}, \tau^{\prime}$ в зонах передруйнування.

Компоненти матриці $\mathbf{N}=\left[N_{i j}\right]_{i, j=1,2}$ мають такий вигляд:

$$
N_{11}=\int_{a_{1}}^{a}\left(\frac{t-b_{1}}{t-a_{1}}\right)^{0,5+i \varepsilon} d t, N_{12}=\int_{b}^{b_{1}}\left(\frac{t-b_{1}}{t-a_{1}}\right)^{0,5+i \varepsilon} d t,
$$


ISSN 2074-5893 Питання прикладної математики і математичного моделювання. Випуск 20

$$
N_{21}=\int_{a_{1}}^{a}\left(\frac{t-a_{1}}{t-b_{1}}\right)^{0,5-i \varepsilon} d t, N_{22}=\int_{b}^{b_{1}}\left(\frac{t-a_{1}}{t-b_{1}}\right)^{0,5-i \varepsilon} d t .
$$

Елементи $N_{i j}$ матриці $\mathbf{N}$ можна знайти чисельно або представити за допомогою гіпергеометричних функцій.

Із системи (30), отримаємо наступні вирази для напружень у зонах передруйнування

$$
\left\{\begin{array}{c}
\sigma^{\prime}+i m_{1} \tau^{\prime} \\
\sigma+i m_{1} \tau
\end{array}\right\}=-\frac{\pi i l_{1}}{1+\gamma_{1}}\left(\sigma^{\infty}+i m_{1} \tau^{\infty}\right) \mathbf{N}^{-1}\left\{\begin{array}{c}
1+2 i \varepsilon \\
-(1-2 i \varepsilon)
\end{array}\right\} .
$$

Після підстановки виразів (32) у рівняння (17), (18) і вибору відповідних значень $\sigma_{1}$ i $\sigma_{1}^{\prime}$, отримуємо систему нелінійних рівнянь

$$
\Psi_{i}\left(\Delta_{a}, \Delta_{b}, \sigma^{\infty}, \tau^{\infty}\right)=0, i=1,2
$$

відносно довжин зон передруйнування

$$
\Delta_{a}=a-a_{1} \text { i } \Delta_{b}=b_{1}-b .
$$

У загальному випадку розв'язки системи (33) можна знайти чисельно.

Для визначення стрибків переміщень використана методика, запропонована в роботі [2].

Згідно з цим підходом отримані наступні вирази для розкриття тріщини в іiї початкових вершинах

$$
\delta_{1}^{a}=\left\langle u_{1}(a)\right\rangle, \delta_{2}^{a}=\left\langle u_{2}(a)\right\rangle, \delta_{1}^{b}=\left\langle u_{1}(b)\right\rangle, \delta_{2}^{b}=\left\langle u_{2}(b)\right\rangle,
$$

які відіграють найбільш важливу роль при використанні деформаційного критерію руйнування. Праві частини формул (35) можуть бути визначені 3 рівняння

$$
\begin{gathered}
\left\langle u_{1}\left(x_{1}\right)\right\rangle+i s_{1}\left\langle u_{3}\left(x_{1}\right)\right\rangle=\frac{1}{2 \pi i \gamma_{1} \vartheta_{1}}\left\{2 \pi i\left(\sigma^{\infty}+i m_{1} \tau^{\infty}\right)\left(x_{1}-a_{1}\right)^{0,5-i \varepsilon}\left(x_{1}-b_{1}\right)^{0,5+i \varepsilon}+\right. \\
\left.+\left(1+\gamma_{1}\right)\left[\left(\sigma^{\prime}+i m_{1} \tau^{\prime}\right) J_{1}\left(x_{1}\right)+\left(\sigma+i m_{1} \tau\right) J_{2}\left(x_{1}\right)\right]\right\}-\frac{1-\gamma_{1}}{2 \gamma_{1} t_{1}} h\left(x_{1}\right) \\
\text { де } \quad J_{1}\left(x_{1}\right) \approx \arccos \left(\alpha_{1}\right) \sqrt{\left(x_{1}-a_{1}\right)\left(b_{1}-x_{1}\right)}+\frac{x_{1}-a}{2} H\left(a_{1}, b_{1}, x_{1}, a\right), \\
H\left(a, b, x_{1}, \xi\right)= \\
\quad J_{2}\left(x_{1}\right) \approx \arccos \left(\alpha_{2}\right) \sqrt{\left(x_{1}-a_{1}\right)\left(b_{1}-x_{1}\right)}-\frac{x_{1}-b}{2} H\left(a_{1}, b_{1}, x_{1}, b\right), \\
=\ln \frac{\left(b-x_{1}\right)\left(x_{1}-a\right)+\left(\xi-x_{1}\right)\left(\frac{a+b}{2}-x_{1}\right)-\sqrt{\left(b-x_{1}\right)\left(x_{1}-a\right)(b-\xi)(\xi-a)}}{\left(b-x_{1}\right)\left(x_{1}-a\right)+\left(\xi-x_{1}\right)\left(\frac{a+b}{2}-x_{1}\right)+\sqrt{\left(b-x_{1}\right)\left(x_{1}-a\right)(b-\xi)(\xi-a)}} \\
\alpha_{1}=\frac{2 a-b_{1}-a_{1}}{l_{1}}, \alpha_{2}=\frac{2 b-b_{1}-a_{1}}{l_{1}} \cdot h\left(x_{1}\right)=\int_{a_{1}}^{x_{1}} p(t) d t
\end{gathered}
$$


На основі формул (35) можна знайти значення

$$
\delta_{a}=\sqrt{\left(\delta_{1}^{a}\right)^{2}+\left(\delta_{2}^{a}\right)^{2}}, \delta_{b}=\sqrt{\left(\delta_{1}^{b}\right)^{2}+\left(\delta_{2}^{b}\right)^{2}},
$$

які можна розглядати як основні параметри руйнування для відповідних вершин тріщини. Після розв'язання рівнянь (33) і визначення положення точок $a_{1}$ i $b_{1}$, електричне та магнітне зміщення можуть бути визначені за допомогою формул (13), (14).

Чисельні результати та їх обговорення. Чисельні експерименти були виконані для біматеріалу, складеного $3 \mathrm{BaTiO}_{3}-\mathrm{CoFe}_{2} \mathrm{O}_{4}$ композитів 3 об'ємним вмістом $\left(V_{f}\right)$ п'єзоелектрика $\mathrm{BaTiO}_{3}$ рівним 0,5 (верхній матеріал) та 0,1 (нижній матеріал) [3]. Відповідні значення фізичних констант біматеріалу наведені в табл. 1. Для розрахунків вибиралось $a=-0,01 \mathcal{M}, b=0,01 \mathcal{M}$, $\sigma^{\infty}=10^{7} \Pi a, \tau^{\infty}=0 \Pi a, D^{\infty}=B^{\infty}=0$.

Таблиия 1

\section{Ефективні властивості ВаTiO$-\mathrm{CoFe}_{2} \mathrm{O}_{4}$ композиту для різних $V_{f}$}

\begin{tabular}{|l|l|l|}
\hline Властивості матеріалів & $V_{f}=0,1$ & $V_{f}=0,5$ \\
\hline$c_{11}($ $П a)$ & 274 & 226 \\
\hline$c_{33}(Г П a)$ & 161 & 124 \\
\hline$c_{13}(Г П a)$ & 259 & 216 \\
\hline$c_{44}(\Gamma П a)$ & 45 & 44 \\
\hline$e_{31}\left(K л / M^{2}\right)$ & $-4,4$ & $-2,2$ \\
\hline$e_{15}\left(K \pi / M^{2}\right)$ & 1,86 & 9,3 \\
\hline$e_{33}\left(K л / M^{2}\right)$ & 1,16 & 5,8 \\
\hline$\alpha_{11}\left(\times 10^{-10} K \pi^{2} / H \cdot M^{2}\right)$ & 11,9 & 56,4 \\
\hline$\alpha_{33}\left(\times 10^{-10} K \pi^{2} / H \cdot M^{2}\right)$ & 13,4 & 63,5 \\
\hline$h_{31}(H / A \cdot M)$ & 522,3 & 290,2 \\
\hline$h_{33}(H / A \cdot M)$ & 629,7 & 350,0 \\
\hline$h_{15}(H / A \cdot M)$ & 495,0 & 275,0 \\
\hline$\mu_{11}\left(\times 10^{-6} H \cdot c^{2} / K \pi^{2}\right)$ & 531,5 & 297,0 \\
\hline$\mu_{33}\left(\times 10^{-6} H \cdot c^{2} / K \pi^{2}\right)$ & 142,3 & 83,5 \\
\hline
\end{tabular}

Міжфазний прошарок вважався ідеально-пружним пластичним матеріалом 3 межею текучості $\sigma_{T}=220$ МПа та умовою текучості Мізеса. Це означає, що функція $f$ в рівняннях (17) і (18) приймається у вигляді

$$
f\left(\sigma, \tau, \sigma_{1}\right) \equiv\left(\sigma-\sigma_{1}\right)^{2}+4 \tau^{2}-4 \sigma_{T}^{2} / 3=0 .
$$


Згідно зі статтею [5], напруження $\sigma_{11}$ на тріщині у згаданому матеріалі рекомендується прийняти рівним $2 \sigma_{T}$. Тому для рівнянь (17), (18) і (32) припускаємо $\sigma_{1}=\sigma_{1}^{\prime}=2 \sigma_{T}$.

Результати розрахунків відносної довжини зони передруйнування, напружень в цій зоні і розкриття тріщини в початковій вершині відносно інтенсивності зовнішнього навантаження представлені в табл. 2.

Таблиия 2

Відносна довжина зони передруйнування, напруження в цій зоні і розкриття тріщини в початковій вершині для біматеріалу PZT4/PZT5 у разі симетричного навантаження

\begin{tabular}{|c|c|c|c|c|c|}
\hline$\frac{\sigma^{\infty}}{\sigma_{T}}$ & $\frac{b_{1}-b}{b}$ & $\frac{\sigma}{\sigma_{T}}$ & $\frac{\tau}{\sigma_{T}}$ & $\frac{\delta_{1}^{b}}{b} \cdot 10^{6}$ & $\frac{\delta_{2}^{b}}{b} \cdot 10^{6}$ \\
\hline 0,05 & 0,0032 & 0,9831 & $-0,0916$ & $-0,5132$ & 0,1159 \\
\hline 0,1 & 0,0126 & 0,9895 & $-0,0725$ & $-0,8326$ & 0,4620 \\
\hline 0,15 & 0,0286 & 0,9925 & $-0,0611$ & $-1,0778$ & 1,0418 \\
\hline 0,2 & 0,0515 & 0,9944 & $-0,0529$ & $-1,2744$ & 1,8624 \\
\hline 0,25 & 0,1405 & 0,9957 & $-0,0465$ & $-1,4369$ & 2,9346 \\
\hline 0,333 & 0,1518 & 0,9971 & $-0,0383$ & $-1,6555$ & 5,3235 \\
\hline
\end{tabular}

Висновки. Запропонована модель зон передруйнування для тріщини на межі поділу п'єзоелектромагнітних матеріалів. Розглянута найбільш адекватна дійсності модель електрично та магнітно проникних умов на берегах тріщини. Поставлену задачу зведено до задачі Гільберта-Рімана з нелінійною залежністю області стрибків невідомої функції від зовнішніх факторів. Знайдені наближені аналітичні залежності довжин зон передруйнування та розкриття тріщини від фізичних характеристик матеріалу і навантаження. Для конкретної комбінації матеріалів продемонстровані числові значення цих залежностей.

\section{Бібліографічні посилання}

1. Лобода, В. В. Межфазная трещина в пьезоэлектромагнитном биматериале под действием механической нагрузки, электрического и магнитного потоков [Текст] / В. В. Лобода, Т. В. Ходанен // Вісник Дніпропетр. ун-ту. Серія: Механіка. - 2007. - Вип. 11, т. 2. C. $117-129$.

2. Loboda, V. Electro-mechanical pre-fracture zones for an electrically permeable interface crack in a piezoelectric bimaterials [Text] / V. Loboda, Y. Lapusta, A. Sheveleva // International Journal of Solids and Structures. - 2007. - Vol.44. - P. 5538-5553. DOI:10.1016/j.ijsolstr.2007.01.013.

3. Sih, G. C. Magnetic and electric poling effects associated with crack growth in $\mathrm{BaTiO}_{3}-$ $\mathrm{CoFe}_{2} \mathrm{O}_{4}$ composite [Text] / G. C. Sih, Z. F. Song // Theoretical and Applied Fracture Mechanics. - 2003. - 39. - P. 209-227.

4. Мусхелишвили, Н. И. Некоторые основные задачи математической теории упругости [Текст]/ Н. И. Мусхелишвили. - М.: Наука. - 1966. - 707 с. 
5. Tvergaard, V. On the toughness of ductile adhesive joints [Text] / V. Tvergaard, J. W. Hutchinson // Journal of the Mechanics and Physics of Solids. - 1996. - Vol. 44. P. 789-800. DOI:10.1016/0022-5096(96)00011-7.

6. Леонов, М. Я. Розвиток найдрібніших тріщин в твердому тілі [Текст] / М. Я. Леонов, В. В. Панасюк // Прикладна механіка. - 1959. - Т. 5, № 4. - С. 391-401.

7. Dugdale, D. S. Yielding of steel sheets containing slits [Text] / D. S. Dugdale // Journal of the Mechanics and Physics of Solids. - 1960. - Vol. 8, № 2. - P. 100-108.

8. Ma, P. Pre-fracture zone model on electrically impermeable and magnetically permeable interface crack between two dissimilar magnetoelectroelastic materials [Text] / P. Ma, W. Feng, R. Su // Eng. Fract. Mech. - 2013. - 102. - P.310-323. DOI:10.1016/j.engfracmech.2013.03.004.

Надійшла до редколегії 20.10.2020. 